\title{
Cost-Effectiveness of Needle and Syringe Exchange for the Prevention of HIV in New York City
}

\author{
Hrishikesh K. Belani \\ Peter A. Muennig
}

\begin{abstract}
Shared needle and syringe use among injection drug users continues to be a major mode of transmission of HIV. Needle and syringe exchange (NSE) may be a viable strategy to reduce the transmission of the virus; yet the difficulty in measuring the actual efficacy of NSE has limited attempts to evaluate the cost-effectiveness of the intervention. Using data specific to the Lower East Side Harm Reduction Center in New York City, we assessed the cost-effectiveness of NSE over a range of conservative estimates of efficacy, obtained from both longitudinal and small-area studies. A decision-analysis model was created to compare the outcomes and costs associated with NSE. Model inputs included the cost of living with HIV and the seroprevalence of HIV among injection drug users in New York City. This analysis was conducted from both the government and societal
\end{abstract}

Hrishikesh K. Belani, MPH, is a consultant with the Lower East Side Harm Reduction Center, New York, NY, USA.

Peter A. Muennig, MD, MPH is Assistant Professor of Health Policy and Management at the Mailman School of Public Health, Columbia University.

Address correspondence to: Hrishikesh K. Belani, 4916 Ridge Road, Edina, MN 55436, USA (E-mail: hkbelani@wustl.edu).

We acknowledge the Lower East Side Harm Reduction Center for providing the funds and the data to complete this study.

Journal of HIV/AIDS \& Social Services, Vol. 7(3) 2008

Available online at http://www.haworthpress.com

(C) 2008 by The Haworth Press. All rights reserved. doi: $10.1080 / 15381500802307492$ 
perspectives. Tested over a range of conservative parameter estimates, NSE appears to save money and lives. The NSE program we evaluated cost $\$ 502$ per client and produced a gain of 0.01 quality adjusted life years per client. It also reduced HIV treatment costs by $\$ 325,000$ per case of HIV averted, and averted 4-7 HIV infections per 1000 clients, producing a net cost savings.

KEYWORDS. Cost-effectiveness analysis, harm reduction, HIV Prevention, needle exchange, needle and syringe exchange, NEP, NSE, NSP, SEP, syringe exchange

\section{BACKGROUND}

More than 25 years after the first cases were reported, HIV/AIDS continues to be a serious health threat in New York City. New York City has the largest AIDS case rate in the country, and HIV/AIDS is the city's third leading cause of mortality for people below the age of 65 (New York City Department of Health and Mental Health Hygiene [NYCDOHMH], 2006a). The New York City Department of Health and Mental Health Hygiene (2006a) estimates the total number of people living with HIV/AIDS in New York City to be between 103,290 and 143,402 people, as of 2002.

Injection drug use continues to be a major mode of transmission in New York City. Seroprevalence among the city's injection drug users (IDUs) - a population of 162,500 individuals - is estimated at approximately 13.5\% (NYCDOHMH, 2004). Between January 1 and March 31, 2005, 16.5\% of persons with a new AIDS diagnoses reported a history of injection drug use (NYCDOHMH, 2004, 2006b).

Needle and syringe exchange (NSE) is a viable strategy to reduce the spread of HIV among IDUs (Des Jarlais et al., 1996, 2005; Hagan, Des Jarlais, Purchase, Reid, \& Friedman, 1995; Hurley, Jolley, \& Kaldor, 1997; Kaplan \& O’keefe, 1993; Laufer, 2001). Needle and syringe exchange programs provide channels for IDUs to exchange used syringes and needles for new, sterile ones, effectively reducing the average circulation time of used, contaminated syringes and needles and lowering the probability of their reuse (Laufer). While NSE is intuitively effective, ethics limit study designs to 
mathematical models, tagged syringe tracking studies, temporal designs, retrospective analyses, and small-area analysis. Needle and syringe exchange is legal in New York State and is regulated by the New York State Department of Health. In New York City there are currently nine legal NSE programs in operation (Harm Reduction Coalition, 2006).

Cost-effectiveness analysis is an important tool for estimating the efficiency of competing alternative medical and health interventions. By providing information on the costs and health benefits of one intervention relative to another, it becomes possible to ascertain how limited funds can be spent to maximize the total number of lives saved and health gained. This study will assess the cost and effectiveness of NSE as an intervention to prevent the transmission of HIV among its clients (relative to no intervention), using cases of HIV averted, as well as the quality adjusted life years (QALYs) gained as outcome measures. A QALY is a year of life lived in a state of perfect health. The QALY simultaneously captures both morbidity and mortality in a single metric (Weinstein, Siegel, Gold, Kamlet, \& Russell, 1996).

The objective of this study is to examine whether NSE programs merit further investment and expansion based on their cost-effectiveness. Because previous studies have found that NSE programs are cost-saving, we biased model inputs against the conclusion that NSE programs will save money, lives, and QALYs (Gold, Gafni, Nelligan, \& Millson, 1997; Laufer, 2001). This approach minimizes uncertainty associated with model outputs. We then tested the model inputs across a broad range of plausible NSE efficacy values in order to ascertain the extent to which NSE programs are cost-saving at the lowest plausible estimates of program efficacy.

This study was commissioned by the Lower East Side Harm Reduction Center (LESHRC), which runs one of the nine NSE programs in operation in New York City. Cost-effectiveness analysis is an important tool for estimating the efficiency of competing alternative medical and health interventions. This study relied on data from the medical literature and anonymous, aggregated administrative data from the Lower East Side Harm Reduction Center. Because no personal information of any individual was accessed or used for this study, Institutional Review Board approval was not required. 


\section{METHODS}

This analysis was conducted from the government perspective using constant 2005 dollars. We constructed a deterministic decisionanalysis model that compared two strategies: participation in NSE (i.e., an IDU chooses to exchange used needles and syringes for new, sterile equipment) versus no participation (i.e., an IDU does not utilize the needles and syringes offered). We excluded costs associated with Hepatitis $\mathrm{B}$ and $\mathrm{C}$ infection, the transmission of which may also be reduced by NSE, biasing the study against the conclusion that needle exchange programs are not cost-effective. Future costs and QALYs were discounted at a rate of 3\% (Weinstein, Siegel, Gold, Kamlet, \& Russell, 1996).

\section{Decision-Analysis Model}

A decision-analysis model was created using TreeAge Pro 6.0 for Microsoft Windows. All assumptions for the model are listed in Table 1. The model is designed to obtain the probabilistic, weighted average cost and effectiveness value of each pathway (NSE or no NSE). The cost, probability of illness, and life expectancy inputs are listed in Table 2. While the NSE pathway is associated with a lower probability of contracting HIV (with its associated costs, changes in health-related quality of life, and longevity), subjects in this arm also incur the costs of NSE. The no-NSE pathway is

TABLE 1. Assumptions Used in Deriving Parameter Estimates and Justification

Assumption

Justification

HIV negative IDUs have a life expectancy similar to populations of the same sociodemographic characteristics

Life expectancy of HIV positive is the same for both NSE and non-NSE pathways

HIV-positive LESHRC clients will incur costs similar to HIV-positive Medicaid patients
No other comparator was readily available, and the baseline life-expectancy value exerts little or no effect on the estimated improvements in life expectancy with SEP

While NSE may improve HIV-positive persons' access to care via medical referrals, this assumption was made to ensure that the results were as conservative as possible

LESHRC survey data reports $75 \%$ of all clients to be enrolled in Medicaid 
TABLE 2. Selected Parameters Included in the Decision-Analysis Model

\begin{tabular}{|c|c|c|c|c|}
\hline Parameter & Base & High & Low & Source \\
\hline $\begin{array}{l}\text { Reduction in HIV } \\
\text { seroprevalence } \\
\text { associated with NSE }\end{array}$ & 0.029 & 0.058 & 0 & $\begin{array}{l}\text { (Des Jarlais et al., } \\
\text { 2005; Hurley, Jolley, } \\
\text { \& Kaldor, 1997) }\end{array}$ \\
\hline $\begin{array}{l}\text { Seroprevalence of HIV } \\
\text { among IDUs in New York } \\
\text { City }\end{array}$ & 0.135 & 0.20 & 0.07 & (New York City, 2004) \\
\hline $\begin{array}{l}\text { Average annual Medicaid } \\
\text { HIV cost per enrollee, } \\
2005 \text { dollars }\end{array}$ & $\$ 34,651.65$ & - & - & $\begin{array}{c}\text { (New York State, } \\
\text { 2001) }\end{array}$ \\
\hline $\begin{array}{l}\text { NSE annual cost per client, } \\
2005 \text { dollars }\end{array}$ & $\$ 501.54$ & - & - & LESHRC \\
\hline $\begin{array}{l}\text { Life expectancy of HIV- } \\
\text { negative IDU, in Quality } \\
\text { Adjusted Life Years } \\
\text { (QALYs) }\end{array}$ & 20.91 & - & - & (Paltiel et al., 2005) \\
\hline $\begin{array}{l}\text { Life expectancy of HIV- } \\
\text { positive IDU, in QALYs }\end{array}$ & 18.34 & - & - & (Paltiel et al., 2005) \\
\hline
\end{tabular}

associated with a higher probability of contracting HIV, but no NSE program costs.

\section{Probabilities}

The reduced probability of HIV transmission through injection drug use associated with NSE was calculated using the seroprevalence of HIV among IDUs and the reduction in seroprevalence associated with NSE programs. The most recent report on HIV prevalence by the NYCDOMH (2004) estimates HIV seroprevalence among IDUs in New York City to be $13.5 \%$ in 2002.

To estimate the efficacy of NSE, we employed a range of possible values, varying from no efficacy to $5.8 \%$ effective at reducing the seroprevalence of HIV. The two best estimates of the reduction in seroprevalence associated with NSEs came from a small-area analysis conducted by Hurley et al. (1997) just before the advent of protease inhibitors, and a prospective study with a before/after control conducted by De Jarlais et al. (2005). The small-area analysis adjusted for relevant covariates and estimated the impact of NSEs on the overall seroprevalence within a geographic region and found that NSEs 
reduced seroprevalence by $5.8 \%$. The latter longitudinal study found that NSE was associated with a 3.3\% reduction in HIV seroprevalence among IDUs in New York City. This study, which spanned from 1990-2002, did not control for the adoption of protease inhibitors in the mid-1990s, nor did it sufficiently control for the effect of the prevention efforts that often accompany NSE, such as condom distribution, in the IDU population under study.

Given the limitations of these studies, and a desire to derive a conservative estimate of NSE efficacy, we arbitrarily chose to halve the value obtained in the Hurley et al. (1997) cross-sectional analysis, yielding a value of $2.9 \%$. This value is lower than the majority of published NSE efficacy values (Des Jarlais et al., 1996, 2005; Hagan et al., 1995; Hurley et al., 1997; Kaplan \& O’keefe, 1993; Laufer, 2001). We created two parameters, $s$, the seroprevalence, and $r$, the reduction in $s$ due to NSE. Using these parameters, we set the probability of HIV transmission for IDUs who do not participate in NSE to be $s$, and the probability of transmission for IDUs who do participate in NSE to be $s /(1+r)$.

\section{Cost}

The costs associated with NSE were obtained for the year 2005 from LESHRC and are listed in Table 2. To ensure that the incremental cost-effectiveness ratio was as conservative as possible, we included both variable and fixed costs, without depreciating fixed assets. Fixed costs constitute $40 \%$ of the LESHRC's budget. Our total estimated costs were therefore substantially higher than previous estimates of costs associated with similar NSEs (Laufer, 2001).

In 2005 LESHRC had 1,484 clients participating in its NSE program. The clients were $72.37 \%$ male, $27.56 \%$ female, and $0.07 \%$ transgender. Seventy-four percent were between the ages 20 and 50, 2\% were below the age of 20 , and $24 \%$ were over the age 50. Approximately $75 \%$ were enrolled in Medicaid. Cost structures for other programs were assumed to be similar.

The costs associated with HIV infection were calculated for life, using life expectancy estimates for an HIV-positive IDU on treatment. As the majority of LESHRC clients are enrolled in Medicaid, the costs associated with HIV infection were obtained from the New York State Department of Health's (2001) 2002 reported annual Medicaid expenditures per enrollee with HIV/AIDS. The 2002 
average Medicaid costs for an enrollee living with HIV/AIDS included inpatient care, ambulatory and emergency room care, freestanding clinic costs, physician costs, pharmacy costs, treatment costs (including antiretrovirals and protease inhibitors), institutional long-term care, home health care, personal care, and other costs (New York State Department of Health). We assumed that the subset of HIV-positive IDUs was mostly receiving Medicaid funding, or funding from other public programs with similar costs.

\section{Life Expectancy}

The average life expectancies for both a HIV-negative and a HIVpositive IDU were obtained from a recent study by Paltiel et al. (2005). Using a computer-simulation model, the researchers estimated mean-quality adjusted survival among high-risk populations in the United States and among HIV-infected members of high-risk populations. These baseline parameter estimates are listed in Table 2 .

\section{Sensitivity Analyses}

Variables $r$ and $s$ were tested for their influence on the model; baseline estimates for these parameters and a range of possible values for each estimate are listed in Table 2. Both variables were tested by univariate analysis, as well as bivariate analysis.

\section{RESULTS}

The cost and effectiveness of each strategy estimated by the decision-analysis model are listed in Table 3. The NSE was the dominant strategy, as it was found to be both cost saving and life saving when $r$ was set to the high value, 0.058 . For year 2005 the program resulted in an estimated savings of about $\$ 3,000$ per client and a gain of 0.02 QALYs due to reduced transmission of HIV, relative to if the program was not in operation. When the baseline value of 0.029 was used, the program resulted in an estimated savings of approximately $\$ 1,300$ per client, a gain of 0.01 QALYs.

Using baseline values, NSE results in a total savings of $\$ 1.3$ million per 1,000 clients, which is the cost associated with approximately four cases of HIV averted, at a cost of $\$ 502$ per client, or $\$ 125,000$ per case of HIV averted. 
TABLE 3. Incremental Cost and Effectiveness of "NSE" and "NO NSE" Strategies

\begin{tabular}{lcccccc}
\hline Strategy & Cost & $\begin{array}{c}\text { Incremental } \\
\text { Cost }\end{array}$ & $\begin{array}{c}\text { Effective- } \\
\text { ness }\end{array}$ & $\begin{array}{c}\text { Incremental } \\
\text { Effective- } \\
\text { ness }\end{array}$ & $\begin{array}{c}\text { Cost- } \\
\text { Effective- } \\
\text { ness }\end{array}$ & $\begin{array}{c}\text { Incremental } \\
\text { Cost- } \\
\text { Effective- } \\
\text { ness Ratio }\end{array}$ \\
\hline NSE & 63,027 & $-1,312$ & 20.57 & 0.01 & 3,064 & - \\
NO NSE & 64,339 & - & 20.56 & - & 3,129 & $\begin{array}{c}\text { (Domi- } \\
\text { nated) }\end{array}$ \\
\hline
\end{tabular}

Note: Because the NSE produced cost-savings, it is the dominant strategy, and an incremental cost-effectiveness ratio is not computed.

The model was tested for the influence of parameters $s$ (HIV seroprevalence among IDUs) and $r$ (the reduction in HIV seroprevalence associated with NSE). The result of a one-way analysis on $r$ is shown in Figure 1. Although $r$ is the most important parameter used in this study, it is also the least reliable. A one-way analysis from 0 (NSE is not effective) to 0.058 (the high value) shows the NSE to be cost saving if it is associated with a reduction in HIV seroprevalence greater than 0.008. Thus, needle exchange is cost saving and life saving at

FIGURE 1. One-way Sensitivity Analysis on NSE-Program Efficacy. The Figure on the Left Shows Costs and Cost-Savings at Various Levels of HIV Seroprevalence Reduction. The Figure on the Right Shows QALY Gains at Various Levels of Seroprevalence Reduction
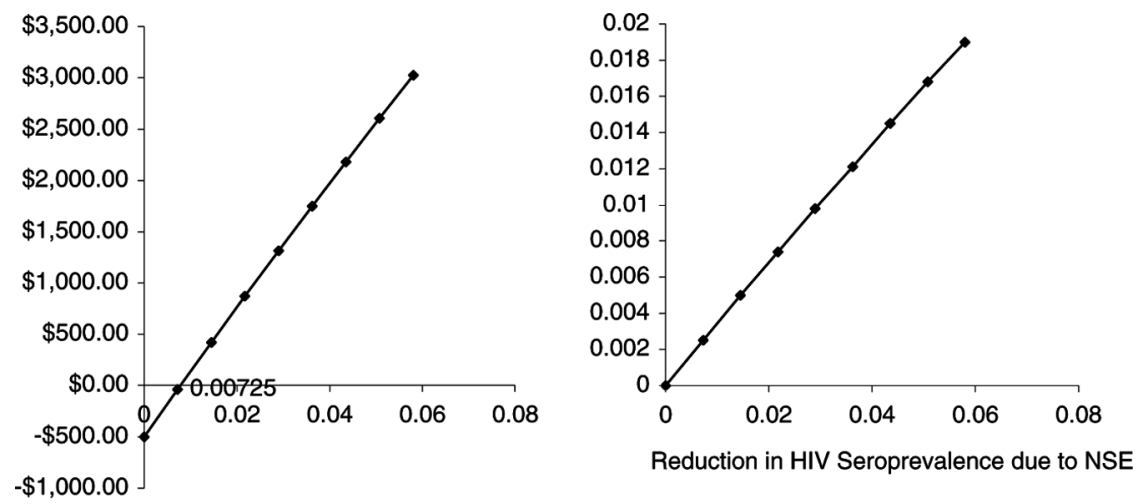

Reduction in HIV Seroprevalence due to NSE 
values less than one-seventh the early estimate obtained from an ecological study.

A one-way sensitivity analysis conducted on $s$, using the NYCDOMH's low and high estimates of HIV seroprevalence among IDUs in New York City, shows the cost-savings associated with the NSE to have a direct linear relationship with $s$. Although the NSE continues to be cost saving across all values of $s$, as $s$ decreases, the cost savings also decrease. The NSE strategy therefore remains dominant over the plausible values of HIV seroprevalence in the IDU population, and of the reduction in HIV seroprevalence associated with NSE.

\section{DISCUSSION}

The LESHRC needle exchange program appears to save both money and lives from both the government and societal perspectives. From the governmental perspective, NSE results in a savings of $\$ 1,300-\$ 3,000$ per client. From the societal perspective, NSE produces a net gain of $0.01-0.02$ QALYs and averts $0.004-0.007$ cases of HIV per client per year.

However, this prediction is contingent on the extent to which the reduction in HIV seroprevalence associated with the NSE was accurately predicted by a single ecological study conducted before the introduction of protease inhibitors into the medical marketplace. Protease inhibitors, which became available at the end of 1995, are now a standard part of HIV treatment (Wynn et al., 2004). Because protease inhibitors act to reduce the amount of HIV virus in the blood, the rate of transmission via shared-syringe use is likely significantly lower compared to what it was during time frame of Hurley et al.'s study (1997), and the reduction in HIV seroprevalence associated with NSE would in effect be lower.

While it is difficult to estimate how much lower infection rates might be, there are a number of reasons to suspect that NSE may be cost saving. First, not all IDUs are receiving protease inhibitors. Second, NSE remains cost-effective even if the actual reduction in seroprevalence among IDUs is 0.008 - about one-fourth the value estimated by Des Jarlais et al. (2005), and one-seventh the value estimated by Hurley et al. (1997). Third, the seropositive estimates of 0.029 and 0.058 reflect overall seroprevalence reduction in the general 
IDU population. Analyses specific to the population within a NSE program would likely be substantially higher since only a fraction of IDUs in a given geographic location actually have access to NSE. Fourth, we excluded important cost-savings associated with NSE, such as the possible reduction of Hepatitis B and Hepatitis C transmission. Fifth, the costs associated with NSE used for this study, $\$ 502$ per client per year, were obtained from a New York City NSE, where the costs of operation are likely higher than in most other parts of the country.

This study has a number of limitations. Foremost, there is no good estimate of NSE efficacy. Our analysis found that NSE was costeffective even when efficacy was one-seventh of the value from a small-area analysis and one-fourth that of a longitudinal study design. Nonetheless, small-area analyses may be confounded by a number of unforeseen ecological confounders, such as program implementation in areas where people are generally receptive to health education programs. Likewise, longitudinal studies are potentially confounded by temporal effects, such as the drop in HIV incidence seen in the United States over time (though incidence outside of the study population was considered in deriving the estimate of NSE efficacy). While such studies find a strong correlation between the number of needles distributed and dropping incidence of HIV/AIDS, AIDS, both can be correlated with other factors. For instance, advances in HIV/AIDS treatments also lower blood levels of HIV virus, and therefore lower transmission rates. One other limitation was our reliance upon modeled data for QALY estimation (Paltiel et al., 2005). This model, while developed by some of the nations' leading HIV/AIDS and modeling experts using the best available data, relies on a number of assumptions that may bias its outputs. Model input error was limited by biasing the study against the conclusion that NSE is cost saving as described above.

Needle and syringe exchange is an effective HIV prevention intervention that can also be a cost saving to Medicaid, as seen by the cases of HIV averted by the Lower East Side Harm Reduction Center in 2005. This finding is pertinent to social service providers, as it can inform the development of a community-level strategy to prevent HIV/AIDS and the decision to devote resources to a NSE program. Needle and syringe exchange programs (mobile or stationary) can also serve as a point of delivery for multiple social services including those directly related to HIV/AIDS prevention (such as behavioral 
and biomedical prevention interventions and referral to care and treatment) and also more general services (such as mental health counseling, general health services, life-skills training, job-assistance, and legal services).

Needle and syringe exchange continues to be a controversial intervention, however, and faces opposition in the United States. While states can legalize NSE programs, they cannot receive federal funding. More comprehensive and up-to-date evaluation of NSE programs would provide evidence of the full benefits of this intervention strategy and will assist these programs in gaining wider acceptance, more funding, and greater capacity.

Nonetheless, this study uses conservative estimates of the efficacy of NSE programs and still produces cost-savings and QALY gains. Policy makers can therefore be reasonably certain that such programs will save lives and money. Because most medical interventions-even most prevention programs - are associated with some net cost, this study suggests that NSE programs should be prioritized for funding.

\section{REFERENCES}

Des Jarlais, D. C., Marmor, M., Paone, D., Titus, S., Shi, Q., Perlis, T., et al. (1996). HIV incidence among injecting drug users in New York City syringe-exchange programmes. Lancet, 348(9033), 987-991.

Des Jarlais, D. C., Perlis, T., Arasteh, K., Torian, L. V., Beatrice, S., Milliken, J., et al. (2005). HIV incidence among injection drug users in New York City, 1990 to 2002: Use of serologic test algorithm to assess expansion of HIV prevention services. American Journal of Public Health, 95(8), 1439-1444.

Gold, M., Gafni, A., Nelligan, P., \& Millson, P. (1997). Needle exchange programs: An economic evaluation of a local experience. Canadian Medical Association Journal, 157(3), 255-262.

Hagan, H., Des Jarlais, D. C., Purchase, D., Reid, T., \& Friedman, S. R. (Eds.). (1995). Preventing HIV transmission: The role of sterile needles and bleach. Washington, DC: National Academy Press/Institute of Medicine.

Harm Reduction Coalition. (2006). Retrieved July 8, 2006, from http://www. harmreduction.org

Hurley, S. F., Jolley, D. J., \& Kaldor, J. M. (1997). Effectiveness of needle-exchange programmes for prevention of HIV infection. Lancet, 349(9068), 1797-1800.

Kaplan, E. H. \& O'keefe, E. (1993). Let the needles do the talking! Evaluating the New Haven needle exchange. Interfaces, 23, 7-26.

Laufer, F. N. (2001). Cost-effectiveness of syringe exchange as an HIV prevention strategy. Journal of Acquired Immune Deficiency Syndrome, 28(3), 273-278. 
New York City Department of Health and Mental Hygiene. (2004). HIV prevalence in New York City, 2002: HIV epidemiology program special supplemental report. Retrieved July 8, 2006, from http://www.nyc.gov/html/doh/downloads/pdf/ dires/dires-2004-report-vol2noS2.pdf.

New York City Department of Health and Mental Hygiene. (2006a). Retrieved July 8, 2006, from http://www.nyc.gov/html/doh/html/ah/ah.shtml

New York City Department of Health and Mental Hygiene. (2006b). HIV Epidemiology program quarterly report. Retrieved July 8, 2006, from http://www.nyc.gov/ $\mathrm{html} / \mathrm{doh} / \mathrm{html} /$ dires/epi_reports.shtml\#quarterly.

New York State Department of Health. (2001). AIDS in New York State. Retrieved May 4, 2006, from http://www.health.state.ny.us/diseases/aids/reports/2001/ index.htm

Paltiel, A. D., Weinstein, M. C., Kimmel, A. D., Seage, G. R., 3rd, Losina, E., Zhang, H., et al. (2005). Expanded screening for HIV in the United States-An analysis of cost-effectiveness. New England Journal of Medicine, 352(6), 586-595.

Weinstein, M. C., Siegel, J. E., Gold, M. R., Kamlet, M. S., \& Russell, L. B. (1996). Recommendations of the panel on cost-effectiveness in health and medicine. Journal of the American Medical Association, 276(15), 1253-1258.

Wynn, G. H., Zapor, M. J., Smith, B. H., Wortmann, G., Oesterheld, J. R., Armstrong, S. C., et al.. (2004). Antiretrovirals, part 1: Overview, history, and focus on protease inhibitors. Psychosomatics, 45(3), 262-270. 